\title{
Clinical diagnosis and treatment of common respiratory tract infections in relation to microbiological profiles in rural health facilities in China: implications for antibiotic stewardship
}

Xingrong Shen ${ }^{1,2}$, Jilu Shen ${ }^{3}$, Yaping Pan ${ }^{4}$, Jing Cheng ${ }^{2}$, Jing Chai ${ }^{2}$, Karen Bowker ${ }^{5}$, Alasdair MacGowan ${ }^{5}$, Isabel Oliver ${ }^{6}$, Helen Lambert ${ }^{7}$ and Debing Wang ${ }^{1,2^{*}}$

\begin{abstract}
Background: This paper tries to describe prevalence and patterns of antibiotics prescription and bacteria detection and sensitivity to antibiotics in rural China and implications for future antibiotic stewardship.

Methods: The study was implemented in one village clinic and one township health center in each of four rural residential areas in Anhui Province, China. It used mixed-methods comprising non-participative observations, exit-survey and microbiological study. Observations were conducted to record clinical diagnosis and antibiotic prescription. Semi-structured questionnaire survey was used to collect patient's sociodemographic information and symptoms. Sputum and throat swabs were collected for bacterial culture and susceptibility testing.

Results: A total of 1068 (51.0\% male vs 49.0\% female) patients completed the study with diagnosis of respiratory tract infection (326,30.5\%), bronchitis/tracheitis (249,23.3\%), pharyngitis (119,11.1\%) and others (374, 35.0\%). They provided 683 sputum and 385 throat swab specimens. Antibiotics were prescribed for $88 \%$ of the RTI patients. Of all the specimens tested, 329 (31\%) were isolated with bacteria. The most frequently detected bacteria were K. pneumonia (24\% in all specimens), H. influenza (16\%), H. parainfluenzae (15\%), P. aeruginosa (6\%), S.aureus (5\%), M. catarrhalis (3\%) and S. pneumoniae (2\%).
\end{abstract}

Conclusions: The study establishes the feasibility of conducting microbiological testing outside Tier 2 and 3 hospitals in rural China. It reveals that prescription of antibiotics, especially broad-spectrum and combined antibiotics, is still very common and there is a clear need for stewardship programs aimed at both reducing the number of prescriptions and promoting single and narrow-spectrum antibiotics.

Keywords: Antibiotic, Respiratory tract infection, Primary care, Diagnosis, Microbiological, China

*Correspondence: dbwang@vip.sina.com

1 School, of Public Health, Anhui Medical University, Hefei, Anhui, China

Full list of author information is available at the end of the article

(c) The Author(s) 2021. Open Access This article is licensed under a Creative Commons Attribution 4.0 International License, which permits use, sharing, adaptation, distribution and reproduction in any medium or format, as long as you give appropriate credit to the original author(s) and the source, provide a link to the Creative Commons licence, and indicate if changes were made. The images or other third party material in this article are included in the article's Creative Commons licence, unless indicated otherwise in a credit line to the material. If material is not included in the article's Creative Commons licence and your intended use is not permitted by statutory regulation or exceeds the permitted use, you will need to obtain permission directly from the copyright holder. To view a copy of this licence, visit http://creativecommons.org/licenses/by/4.0/. The Creative Commons Public Domain Dedication waiver (http://creativeco mmons.org/publicdomain/zero/1.0/) applies to the data made available in this article, unless otherwise stated in a credit line to the data. 


\section{Background}

Antimicrobial resistance (AMR) has become one of the biggest threats to global health $[1,2]$. It leads to higher financial costs, prolonged hospital stays and increased patient mortality. Data from 76 countries including China show that global antibiotics consumption grew by more than 39\% between 2000 and 2015 and China consumes the second largest amount of antibiotics in the world [3] with a prescription rate twice that recommended by the World Health Organization (WHO). Rural areas have higher antibiotic prescribing rates than urban areas [4]. According to a survey in rural areas of Shandong and Ningxia, $89 \%$ and $77 \%$ of the prescriptions contained antibiotics for patients clinically diagnosed as having upper respiratory tract infections (RTIs) [5]. Another survey of prescriptions from village clinics in middle-east China showed that the proportion of antibiotics prescribed for RTIs accounted for $87 \%$ [6]. The Chinese government has introduced a number of regulations to control antibiotic use in the last decade, but these have not played a significant role in rural areas [7].

The majority of people in China live in rural and township areas. Physicians in these areas mainly provide two kinds of services for residents, the treatment of common infectious diseases (mainly RTIs), and the management of chronic diseases. The excessive antibiotics prescriptions mostly occur in treating common RTIs. A range of possible reasons for unnecessary use of antibiotics by rural and township physicians have been identified. It is not uncommon for them to prescribe antibiotics 'just in case', due to a desire to reduce medical, legal and reputation risk in the face of diagnostic uncertainty [8]. Antibiotics may also be used unnecessarily under the driver of income interests, including prescribing of inappropriate antibiotics for particular diseases, unnecessary escalation (for example prescribing more expensive and broadspectrum antibiotics when cheaper and more specific antibiotics can give the same result) and intravenous use $[9,10]$. In addition, most rural and township physicians in China have to distinguish bacterial or virus infection based on patient-reported symptoms because microbiological facilities are not available at most front-line health care settings [11].

Most research on AMR in China has drawn on patient data collected in urban specialist hospitals. The often asserted high burden of AMR in China may be misleading since current assumptions are based on potential pathogens isolated in selected microbiology laboratories enrolled in the national surveillance system and it is uncertain if this reflects the incidence of resistance in non-hospitalized patients with mild to moderate infection receiving antibiotics, most of whom do not have samples sent to the laboratory $[12,13]$. UK data indicates that 1:35 patients presenting to their general practitioners with acute cough have microbiological sampling; while the same sample proportion is only about 1:5 at secondary and tertiary medical care settings [14]. These data indicate that resistance rates to key antibiotics based on biased laboratory data may be over-emphasized [1518]. Similarly, the often reported reduction in antibiotics prescription rate derived from paper or electronic medical records may also be misleading since such records in most primary clinics in China are incomplete or inaccurate and the bulk of antibiotics is used at primary care settings.

This paper tries to describe prevalence and patterns of antibiotics prescription and bacteria detection and sensitivity to antibiotics in rural China and implications for future antibiotic stewardship. It uses data from a mixed methods project co-sponsored by China National Natural Science Foundation and UK Research and Innovation [14].

\section{Methods}

This study is part of the "pathways to optimizing antibiotic use in Anhui: identifying key determinants in community and clinical settings' project. It used mixed-methods consisting of qualitative interviews, non-particative observations, semi-structured surveys and microbiological studies. The study protocol has been published separately [14]. This paper used mainly the quantitative data from the study.

\section{Setting}

The study was implemented in one village clinic and one township health center in each of four rural residential areas in Anhui Province, China. Anhui Province is located in the middle east of China and has a population of 68.6 million of whom $57 \%$ live in rural areas. It has 968 hospitals, 1,941 community and 1,398 township health centers, and 15,288 village clinics.

\section{Participants}

The study aimed to recruit 1,000 patients presenting with respiratory infection [14]. Inclusion criteria were male or female patients who were: a) 18 years or older and able to give consent to participate in the microbiological study and exit survey; b) presenting to the recruitment site for his/her current illness for the first time during the study period; and c) observed as having one or more of the following: exacerbation of chronic obstructive pulmonary disease (COPD), upper respiratory tract infection with productive cough or sore throat. Selection of these conditions was based on the consideration that there may be differences between patients with different clinical 
diagnosis (e.g., higher chances for identifying bacteria and antibiotics resistance among patients with exacerbation of COPD than those with cough and/or a sore throat). Acute otitis and rhinosinusitis were not included because these diseases happen mainly in children while this study population comprised only adults (over 18-year-old).

Patients were selected via "consecutive sampling" in which, when a start date had been determined, the recruitment continued daily (7 days a week) thereafter, between $8 \mathrm{am}-5 \mathrm{pm}$ or $9 \mathrm{am}-6 \mathrm{pm}$ on alternate days, until the target numbers had been reached. All incoming patients to the site village clinics and township health centers who met the inclusion criteria during any study day were invited to participate. This was a pragmatic approach to sampling since patient record systems do not allow the flexibility to carry out random sampling, and ongoing recruitment ensured the most efficient use of staff and resources in this setting.

\section{Data collection}

\section{Semi-structured observations and exit surveys}

A trained researcher was sent to each participating clinics and health centers to perform semi-structured observations and exist survey. The observation focused on daily operational routine including test ordering, prescribing, patient recall and other standard procedures using a pre-designed worksheet [14]. The exit survey used a questionnaire consisting of structured and semistructured questions informed by open-ended interviews undertaken in the study's pilot phase and included information on social demographics, symptoms and diseases history. The questionnaire was administered face-to-fact by the researcher for all patients consented by the attending clinicians at clinics and health centers and recruited into the study. The questionnaire was completed when the episode of care had concluded.

\section{Specimen collection and microbiological testing}

The study collected sputum and throat swabs for bacterial culture, identification and susceptibility testing. Sputum was collected from patients presenting with productive cough and throat swabs from patients with sore throat. Samples were collected by the attending doctor using a sterilized container and according to a standard protocol. The specimens were transported and tested at the Central Laboratory of Anhui Medical University (AMU). For details of the test procedures, please refer to our published protocol [14].

\section{Data process and analysis}

Recordings from the semi-structured observations were examined to see whether and which antibiotics were prescribed for the symptomatic RTI patients. Two researchers performed the examination independently and discordances were solved by discussions between them. For data from the semi-structured exit-survey, only the structured items were used in this study. This paper reports on descriptive analysis of: a) social demographics (sex, age and educations) of patients recruited; b) antibiotics use by clinical diagnosis; c) percentages of patients identified with specific types of bacteria by groups of clinical diagnosis; and d) prevalence rates of resistance of most frequently identified bacteria to commonly used antibiotics.

\section{Results}

\section{Social demographics of informants}

A total of 1073 patients meeting our inclusion criteria were invited to participate and 1068 (51\% males and 49\% females) provided specimens and completed the face-toface survey. Of these, 683 provided sputum and 385 provided throat swab specimens. Over $60 \%$ of their visits to the clinics or health centers happened within 4 days after onset of infection symptoms and the mean time interval was 6 days. Over half (55\%) of the patients had less than 5 years of education with $26 \%$ being illiterate. There were statistically significant differences between sex groups with males being older and more educated (Table 1).

\section{Clinical diagnosis and antibiotic use}

Antibiotics were prescribed for $88 \%$ of all recruited patients and $36 \%$ of these prescriptions contained two or

Table 1 Socio-demographic characteristics of respondents

\begin{tabular}{clll}
\hline & \multicolumn{2}{l}{ Type of specimens } & Total \\
\cline { 2 - 3 } & Sputum & Throat swab & \\
\hline Age & & & \\
$<=39$ & $124(18.2)$ & $148(38.4)$ & $272(25.5)$ \\
$40-53$ & $168(24.6)$ & $114(29.6)$ & $282(26.4)$ \\
$54-64$ & $183(26.8)$ & $69(17.9)$ & $252(23.6)$ \\
$>=65$ & $208(30.5)$ & $52(13.5)$ & $260(24.3)$ \\
Missing & $0(0.0)$ & $2(0.5)$ & $2(0.2)$ \\
Education & & & \\
0 & $211(30.9)$ & $65(16.9)$ & $276(25.8)$ \\
$1-5$ & $211(30.9)$ & $95(24.7)$ & $306(28.7)$ \\
$6-8$ & $121(17.7)$ & $108(28.1)$ & $229(21.4)$ \\
$>8$ & $134(19.6)$ & $116(30.1)$ & $250(23.4)$ \\
Missing & $6(0.9)$ & $1(0.3)$ & $7(0.7)$ \\
Sex & & & \\
Male & $366(53.6)$ & $179(46.5)$ & $545(51.0)$ \\
Female & $317(46.4)$ & $206(53.5)$ & $523(49.0)$ \\
Total & $683(64.0)$ & $385(36.0)$ & $1068(100.0)$ \\
\hline
\end{tabular}


Table 2 Antibiotic use by clinical diagnosis, N (\%)

\begin{tabular}{|c|c|c|c|c|c|c|c|c|c|}
\hline & \multirow[t]{2}{*}{ Patients } & \multirow[t]{2}{*}{ Any antibiotic } & \multicolumn{3}{|c|}{ Number of antibiotics used } & \multicolumn{4}{|c|}{ Category of antibiotics used } \\
\hline & & & 1 & 2 & $3+$ & Quinolones & Cephalosporins & Penicillins & Others \\
\hline \multicolumn{10}{|l|}{ Diagnosis } \\
\hline -Respiratory tract infection & $326(30.5)$ & $296(90.8)$ & $179(54.9)$ & $92(28.2)$ & $24(7.4)$ & $112(34.4)$ & $92(28.2)$ & $141(43.3)$ & $22(6.7)$ \\
\hline -Bronchitis/tracheitis & $249(23.3)$ & $234(94.0)$ & $109(43.8)$ & $101(40.6)$ & $23(9.2)$ & $106(42.6)$ & $82(32.9)$ & $109(43.8)$ & $7(2.8)$ \\
\hline -Pharyngitis & $119(11.1)$ & $104(87.4)$ & $84(70.6)$ & $15(12.6)$ & $4(3.4)$ & $17(14.3)$ & $20(16.8)$ & $54(45.4)$ & $10(8.4)$ \\
\hline -Common cold & $85(8.0)$ & $69(81.2)$ & $57(67.1)$ & $7(8.2)$ & $5(5.9)$ & $12(14.1)$ & $50(58.8)$ & $11(12.9)$ & $5(5.9)$ \\
\hline $\begin{array}{l}\text {-Pneumonia/bronchopneu- } \\
\text { monia }\end{array}$ & $48(4.5)$ & $44(91.7)$ & $22(45.8)$ & $16(33.3)$ & $6(12.5)$ & $16(33.3)$ & $25(52.1)$ & $17(35.4)$ & $2(4.2)$ \\
\hline -Tonsillitis & $44(4.1)$ & $41(93.2)$ & $19(43.2)$ & $15(34.1)$ & $7(15.9)$ & $18(40.9)$ & $17(38.6)$ & $18(40.9)$ & $1(2.3)$ \\
\hline$-C O P D$ & $29(2.7)$ & $26(89.7)$ & $4(13.8)$ & $15(51.7)$ & $7(24.1)$ & $20(69.0)$ & $7(24.1)$ & $17(58.6)$ & $0(0.0)$ \\
\hline -Others & $24(2.2)$ & $15(62.5)$ & $11(45.8)$ & $4(16.7)$ & $0(0.0)$ & $4(16.7)$ & $7(29.2)$ & $5(20.8)$ & $1(4.2)$ \\
\hline -Diagnosis not given & $144(13.5)$ & $109(75.7)$ & $71(49.3)$ & $31(21.5)$ & $7(4.9)$ & $30(20.8)$ & $59(41.0)$ & $32(22.2)$ & $15(10.4)$ \\
\hline$P$ & & 0.000 & 0.000 & 0.000 & 0.002 & 0.000 & 0.000 & 0.000 & 0.015 \\
\hline Total & $1068(100)$ & $938(87.8)$ & $556(52.1)$ & $296(27.7)$ & $86(8.1)$ & $335(31.4)$ & $359(33.6)$ & $404(37.8)$ & $63(5.9)$ \\
\hline
\end{tabular}

more types of antibiotics (Table 2). The most common clinical diagnosis were "respiratory tract infection"(31\%), "bronchitis/tracheitis" (23\%), "pharyngitis"(11\%), "common cold"(8\%), "pneumonia/bronchopneumonia" (5\%) and "tonsillitis"(4\%). Patients diagnosed with "bronchitis/ tracheitis" witnessed the highest antibiotics prescription rate (94\%), followed by "tonsillitis" (93), "pneumonia/ bronchopneumonia" (91\%) and "respiratory tract infection" (90\%). Prescription of multiple (2 or more) antibiotics happened most frequently for COPD (76\%), bronchitis/tracheitis (50\%), tonsillitis (50\%) and pneumonia/ bronchopneumonia (46\%) patients. The most commonly used antibiotics were penicillins, cephalosporins and quinolones. In terms of the anatomical therapeutic chemical (ATC) classification, J01MA12 ranked the highest (31\% in all the patients studied), followed by J01CA04 (15\%), J01FA13 (15\%), J01CR02 (15\%) and J01DC02 (10\%) (Additional file 1).

\section{Diagnosis and bacteria identification}

Of all the specimens tested, 329 (31\%) were isolated with pathogenic bacteria or asymptomatic carrier, and 23 of them, with two strains of bacteria. These 352 isolates comprised 85 (24\%) for K. pneumonia, 57 (16\%) for $H$. influenzae, 52(15\%) for $H$. parainfluenzae, 22(6\%) for $P$. aeruginosa, 18 (5\%) for S.aureus, 12 (3\%) for M. catarrhalis, 8 (2\%) for S. pneumoniae, 6 (1\%) for $\beta$.haemolytic streptococci, 5(1\%) for A.baumannii and 4(1\%) for E.coli. The study did not find statistically significant difference in the chances of bacteria isolation between patients with different clinical diagnosis. Table 3 provides bacterial detection results from sputum and throat swab specimens by diagnosis; while the results from sputum and throat swab specimens are given separately in Additional files 2 and 3.

\section{Antibiotics prescription and bacteria sensitivity}

Figure 1 portraits the prescription rate of specific antibiotics and resistance rate of the top 5 bacteria isolated from sputum and swab specimens. These bacteria were tested sensitive to most of the antibiotics studied. The highest resistance rate was estimated as $97.4 \%$ for K. pneumoniae to Ampicillin followed by 92.3 for S. aureus to Penicillin and $62.3 \%$ for H.influenzae to Sulfamethoxazole. No clear association between the frequency of antibiotics prescription and the rates of resistance was observable. The highest resistance rate to the five most frequently prescribed antibiotics was $30.0 \%$.

\section{Discussion \\ Key findings}

This study reveals useful findings on antibiotic use and microbiological test results for patients presenting RTIs symptoms at rural and township care settings in Anhui, China. It documents an antibiotic prescription rate as high as $87.8 \%$ and most frequent use of broad-spectrum and multiple antibiotics. It demonstrates that nearly one third $(30.8 \%)$ of the specimens were isolated with pathogenic bacteria or asymptomatic carrier with the top bacteria strains being $K$. pneumonia, $H$. influenza, $H$. parainfluenzae, $P$. aeruginosa and $S$. aureus and the highest resistance rate being $K$. pneumoniae to ampicillin followed by S.pneumoniae to Clindamycin, $H$. influenzae to Trimethoprim/ Sulfaisoxazole and $H$. parainfluenzae to Ampicillin. 


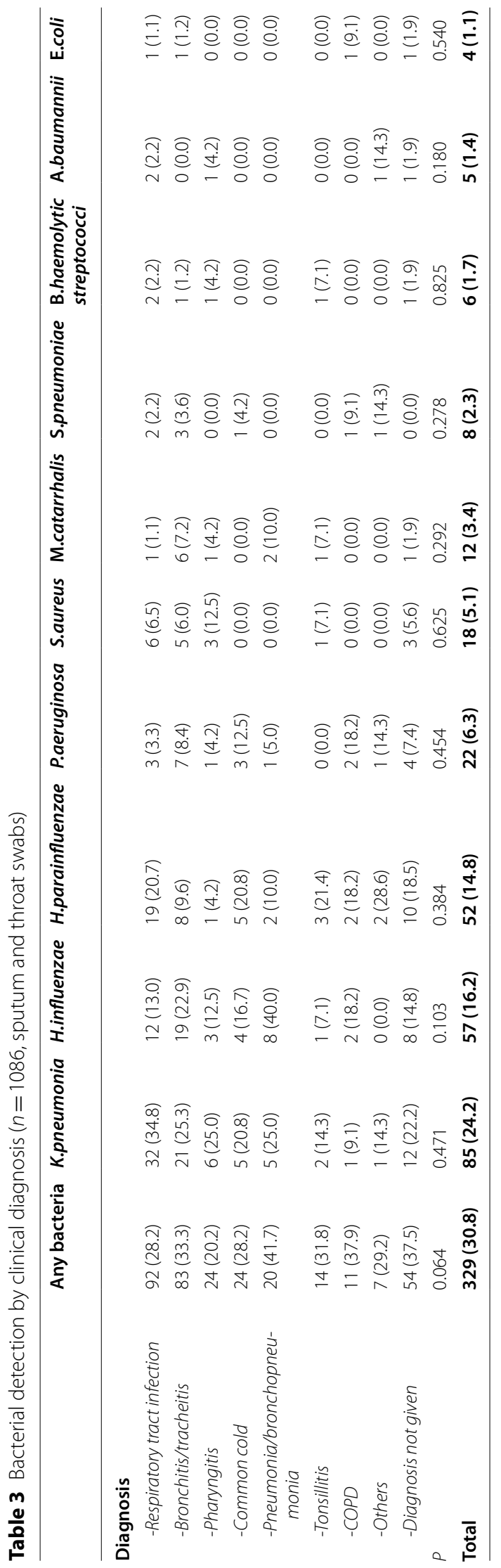




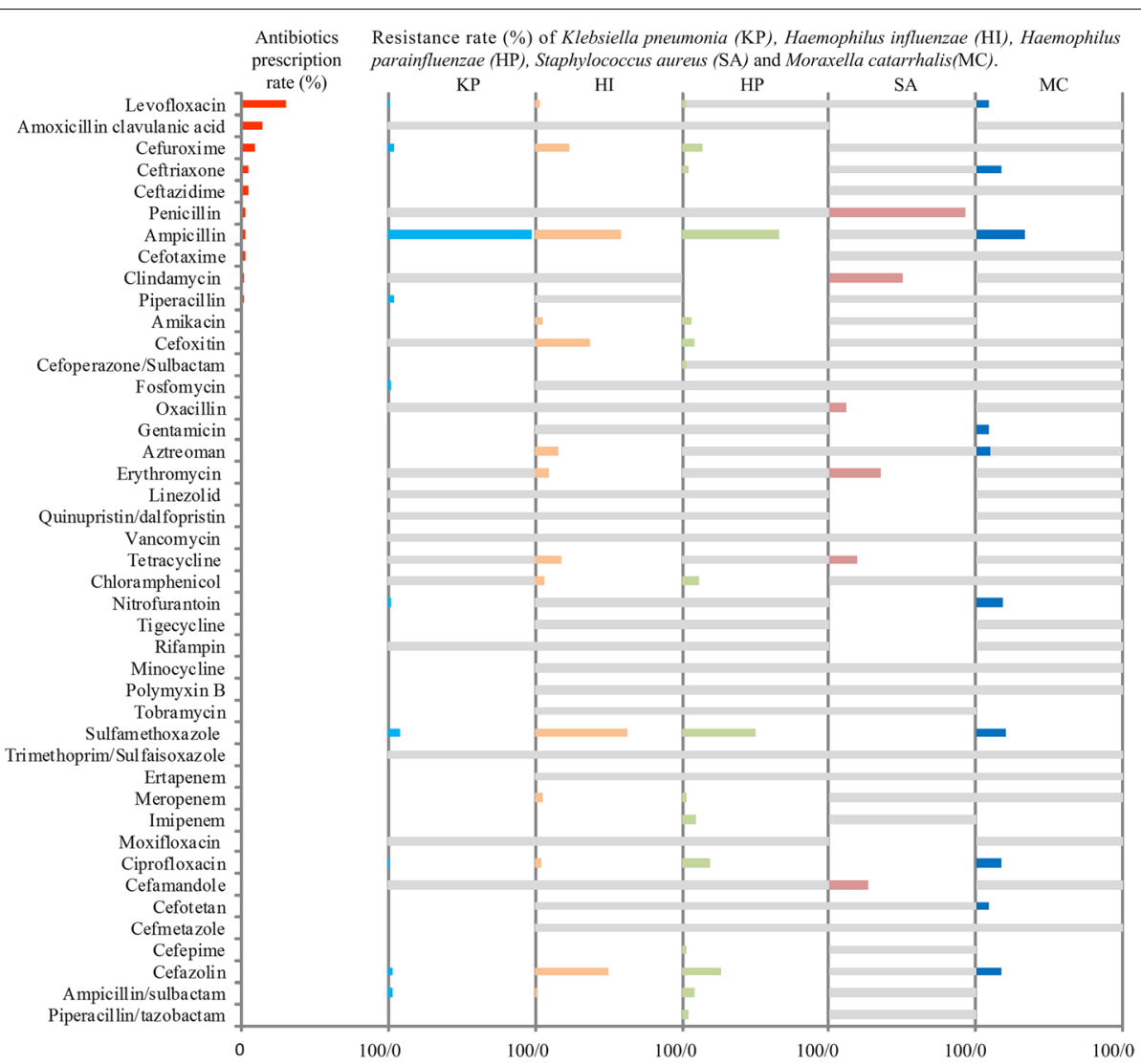

Fig. 1 Antibiotics prescription rates compared with antibiotics resistance rates (grey bars represent not applicable or not performed tests)

Implications in context of other research and for policy The above study findings have important implications for antibiotics stewardship. The high rate of antibiotics prescriptions contradicts a common belief among policymakers in China that excessive antibiotics use is being brought under control as a result of the nationwide Special Antibiotics Use Rectification program (initiated in 2011) and the New Health System Reforms $[19,20]$. These initiatives focus on antibiotics use at secondary and tertiary hospitals. Given that about 57\% of China's vast population lives in rural and township areas and over $70 \%$ of antibiotics prescriptions occur at settings in these areas [21,22], there is a clear need for added attention on excessive antibiotics use at these settings and communities.

The frequent use of broad-spectrum and multiple ( 2 or more) antibiotics calls for stewardship programs aiming at not only reducing the number of prescriptions but also promoting single and narrow-spectrum antibiotics. Most of the broad-spectrum antibiotics, especially quinolones, cephalosporins and amoxi-clav, have strong resistance driving effect; while use of multiple antibiotics often acts as an even greater driver. This prevalent use of broad-spectrum and multiple antibiotics may be attributed mainly to medical uncertainty though decisions on which specific antibiotics to use depends on a variety of factors including availability, price, sensitivity, adverse effects and other characteristics of the antibiotics under concern [23-27]. Rural and township healthcare doctors in China work in a difficult situation in which microbiological tests are unavailable and it is hard to tell the pathogen and its sensitivity to specific antibiotics from clinical symptoms/ history. So, they tend to view broad-spectrum or combined antibiotics as a safer strategy than narrow spectrum antibiotics since the former have greater chance of hitting the actual pathogen $[28,29]$.

The microbiological test results indicate the feasibility of such tests for rural and township care attendees in resource-poor rural China. As described in our separate protocol paper, the testing proceeded by collecting specimens at the rural and township care settings and sending the specimens to a tertiary hospital with a microbiological lab via existing transportation 
services [14]. We collected and tested 1068 specimens out of 1073 RTI patients. Our overall rate of bacteria detection was $30.8 \%$ which is compatible with published results for similar population groups [30]. These all suggest that the testing is acceptable to both patients and physicians and the test results are relatively reliable.

The difference between the resistance rates tested in this study and that from higher level settings suggests a need for incorporating rural and township care settings into China's national antibiotics use and resistance surveillance systems. Our study indicates that antibiotics resistance rates among rural and township care attendees are substantially lower than that among patients of hospitals forming the national antibiotics resistance surveillance network, being $2.5 \%$ vs $56.6 \%$ for K.pneumonia to cefazolin, $58.0 \%$ vs $60.3 \%$ for H.influenzae to ampicillin, $5.9 \%$ vs $25.2 \%$ for P.aeruginosa to piperacillin, $35.3 \%$ vs $61.5 \%$ for S.aureus to erythromycin and $80.0 \%$ vs $89.9 \%$ for S.pneumoniae to clindamycin [30]. These differences in resistance between hospitals and primary care would imply lower general antibiotics prescribing, and especially more narrow-spectrum antibiotics. However, as mentioned above, doctors at primary care settings are practicing the opposite.

The early healthcare seeking together with the high rate of antibiotics prescription highlights the importance of educating doctors and patients to postpone antibiotics prescription and use. The study found that over $60 \%$ of visits to the village clinics or health centers happened within 4 days after onset of infection symptoms and the mean time of visits was 6 days. This is relatively short as compared to that from western nations. Studies from Europe of lower RTIs have documented a mean time lag of 4 to 12 days [31]. Early visits to clinics with a clear expectation to get antibiotics merits adequate attention in future interventions.

Both the profiles of detectable pathogen and asymptomatic carrier bacteria have important implications. Detection of pathogen bacteria (e.g., S.aureus, M.catarrhalis, S.pneumoniae,Bhaemolytic streptococci and E.coli) should inform clinical treatment decisionmaking. Although detection of the asymptomatic carrier may not necessarily cause the infections, it may be used as indirect or surrogate indicators for assessing antibiotic resistance. Perhaps the biggest barrier to microbiological evidence-based antibiotics use for primary care doctors at present may be the delay due to sending specimens to and getting results from higher level labs in addition to time needed for bacteria cultivation and test. Point of care tests may help overcome this barrier. Unfortunately, such tests are generally not available in China [32]. This lack of external validity and clinical uncertainty in primary care together with the links between antibiotics prescription and the number of days until care seeking call for more patient education and more use of a delayed prescribing strategy.

\section{Strengths and limitations of the study}

This study has both strengths and limitations. It is the first study that collected data from healthcare providers and users via a non-participative observation whilst most of the existent research on antibiotics use in China uses data from medical records or reports by medical care givers who may be incentivized to omit recording overuse or misuse of antibiotics so as to meet relevant policy requirements. It is also the first study that performed both microbiological testing and clinical data collection at rural and township care settings and thus enables cross-linking between data from different sources. However, the study suffers from limited number of patients and site clinics. It involved only 8 site clinics or health centers from a single province. So, readers are cautioned about the generalization of the findings to other parts of China, thought the social, cultural and economic background of Anhui is similar to the majority of areas in the nation. The non-participative observation may also have intervened, to some extent, the routine encounters between the patients and doctors and the prescription behaviors being observed though we had arranged a two-week preparation for each site clinic to allow the field researchers to build trust with the doctors. The paper tried to use pure clinical diagnoses to evaluate the other findings; while in reality, it is generally hard to separate the diagnoses of respiratory tract infection and common cold, furthermore pharyngitis and tonsillitis.

\section{Conclusions}

The study makes innovative use of observations in collecting data about antibiotics prescription at rural and township care settings in China for patients with respiratory tract infections and establishes the feasibility of conducting microbiological testing outside Tier 2 and 3 hospitals in rural China. It reveals that prescription of antibiotics, especially broad-spectrum and combined antibiotics, is still very common in rural China and there is a clear need for stewardship programs aimed at both reducing the number of prescriptions and promoting single and narrow-spectrum antibiotics. It also documents important differences in resistance between hospitals and primary care and calls for new specific guidelines for primary care based 
on findings from this and other papers and international expertise like that from the General Practice Respiratory Infections Network.

\section{Abbreviations}

AMR: Antimicrobial resistance; COPD: Chronic obstructive pulmonary disease; RTIs: Respiratory tract infections; AMU: Anhui Medical University; OR: Odds ratio.

\section{Supplementary Information}

The online version contains supplementary material available at https://doi. org/10.1186/s12875-021-01448-2.

\section{Additional file 1.}

Additional file 2 .

Additional file 3.

\section{Acknowledgements}

We would like to thank all the doctors who had helped in the patient recruitment and specimen collection and all the graduate students who had participated in the exit surveys, specimen transportation and data input and cleaning.

\section{Authors' contributions}

XRS directed study implementation and data collection, conducted data analysis and drafted the manuscript. DBW conceptualized and supervised the study together with HL. JLS and YPP performed bacterial culture and drug sensitivity testing. KB and AMG provided expertise on microbiological studies and revised the manuscript together with IO. JC1 and JC2 developed data collection materials and collected data together with XR Shen. The author(s) read and approved the final manuscript.

\section{Funding}

This study was supported by the Newton Fund (UK Research and Innovation (UKRI) and the National Natural Science Foundation of China (NSFC, grant number 81661138001 and 81861138049 ) under the UK-China Antimicrobial resistance Partnership Initiative, grant number MR/P00756/1. The funding source has not plaid any role in the study design, analysis or in the decision to submit the manuscript for publication.

\section{Availability of data and materials}

Data are available from the University of Bristol or Anhui Medical University Data Access (contact via H.Lambert@bristol.ac.uk/dbwang@vip.sina.com) for researchers who meet the criteria for access to confidential data.

\section{Declarations}

Ethics approval and consent to participate

The study was approved by the Biomedical Ethics Committee of Anhui Medical University (reference number: 20170271). The patients and village doctors participated voluntarily, and written informed consent was obtained from all participants.

\section{Consent for publication}

Not applicable.

\section{Competing interests}

The authors declare no potential conflicts of interest with respect to the research, authorship, and/or publication of this article.

\section{Author details}

'School, of Public Health, Anhui Medical University, Hefei, Anhui, China. ${ }^{2}$ School of Health Service Management, Anhui Medical University, Hefei, Anhui, China. ${ }^{3}$ Department of Clinical Laboratory, the Fourth Affiliated
Hospital of Anhui Medical University, Hefei, Anhui, China. ${ }^{4}$ Department of Clinical Laboratory, the First Affiliated Hospital of Anhui Medical University, Hefei, Anhui, China. ${ }^{5}$ Infection Sciences, Severn Pathology, North Bristol NHS Trust, Pathology Building, Southmead Hospital, Westbury-On-Trym, Bristol BS10 5NB, UK. ${ }^{6}$ Field Service, National Infection Service, Public Health England, 3rd floor, 2 Rivergate, Bristol BS1 6EH, UK. ${ }^{7}$ Bristol Medical School, University of Bristol, Bristol, UK.

Received: 2 Auqust 2020 Accepted: 29 April 2021

Published online: 06 May 2021

\section{References}

1. Laxminarayan R, Matsoso P, Pant S, et al. Access to effective antimicrobials: a worldwide challenge. Lancet. 2016;387(10014):168-75.

2. Lim C, Takahashi E, Hongsuwan M, et al. Epidemiology and burden of multidrug-resistant bacterial infection in a developing country. Elife. 2016;5:e18082.

3. Klein EY, Van Boeckel TP, Martinez EM, et al. Global increase and geographic convergence in antibiotic consumption between 2000 and 2015. Proc Natl Acad Sci USA. 2018;115(15):E3463-70.

4. Li Y, Xu J, Wang F, et al. Overprescribing in China, driven by financial incentives, results in very high use of antibiotics, injections, and corticosteroids. Health Aff. 2012;31(5):1075-82.

5. Yin J. Study on drug use in rural area, Shandong province and Ningxia Autonomous Region. Jinan: Shandong University; 2009.

6. Sun Q, Dyar OJ, Zhao L, et al. Overuse of antibiotics for the common cold - attitudes and behaviors among doctors in rural areas of Shandong Province China. BMC Pharmacol Toxicol. 2015;16:6.

7. Xiao Y, Wang J, Shen P, et al. Retrospective survey of the efficacy of mandatory implementation of the essential medicine policy in the primary healthcare setting in China: failure to promote the rational use of antibiotics in clinics. Int J Antimicrob Agents. 2016;48(4):409-14.

8. Shen X, Lu M, Feng R, et al. Web-based just-in-time information and feedback on antibiotic use for village doctors in Rural Anhui, China: randomized controlled trial. J Med Internet Res. 2018;20(2):e53.

9. Zhao J, Liu A, Wen XJ. Outpatient antimicrobial drug in rural grassroots medical institutions outpatient antimicrobial drug use survey in a district of northern. Mod Prev Med. 2016;43(24):4477-80.

10. He AJ. The doctor-patient relationship, defensive medicine and overprescription in Chinese public hospitals: evidence from a cross-sectional survey in Shenzhen city. Soc Sci Med. 2014;123:64-71.

11. Currie J, Lin W, Meng J. Addressing antibiotic abuse in China: an experimental audit study. J Dev Econ. 2014;110:39-51.

12. Yin J, Dyar OJ, Yang P, et al. Pattern of antibiotic prescribing and factors associated with it in eight village clinics in rural Shandong Province, China: a descriptive study. Trans R Soc Trop Med Hyg. 2019;113:trz058.

13. Al-Homaidan HT, Barrimah IE. Physicians' knowledge, expectations, and practice regarding antibiotic use in primary health care. Send Int J Health Sci. 2018;12(3):18-24.

14. Zhao L, Kwiatkowska R, Chai J, et al. Pathways to optimising antibiotic use in rural Anhui province, China: identifying key determinants in community and clinical settings, a mixed methods study protocol. BMJ Open. 2019;9(8):e027819.

15. Cui D, Liu X, Hawkey $P$, et al. Use of and microbial resistance to antibiotics in China: a path to reducing antimicrobial resistance. J Int Med Res. 2017:45(6):1768-78.

16. Tang $X$, Zhuo $C, X u Y C$, et al. The composition and antimicrobial resistance of isolates from lower respiratory 11 tract and blood in hospitalized patients in respiratory ward: a multicenter national study in China. Chinese J Tuberc Respir Dis. 2018;41(4):281-7.

17. McNulty C, Thomas M, John R, et al. Problems of basing patient recruitment for primary care studies on routine laboratory submissions. J Clin Pathol. 2007:60(11):1290-3.

18. Chin TL, MacGowan AP, Bowker KE, et al. Prevalence of antibiotic resistance in Escherichia coli isolated from urine samples routinely referred by general practitioners in a large urban centre in south-west England. J Antimicrob Chemother. 2015:70(7):2167-9. 
19. Hou D, Wang Q, Jiang C, et al. Evaluation of the short-term effects of antimicrobial stewardship in the intensive care unit at a tertiary hospital in China. PLoS ONE. 2014;9:e101447.

20. $\mathrm{Bq} \mathrm{H}$. Effect of special rectification program of inpatients antimicrobials use. Henan J Prev Med. 2015;26:241-3.

21. Shu W, Ren SH, Jiang J, et al. Antibiotics use in five hospitals in our district before and after the implementation of the stewardship strategies for the clinical antimicrobials use. Chinese Pharm. 2015:24:2314-7.

22. National Bureau of Statistics of the People's Republic of China. Bulletin of the sixth national population census data of 2010 in Anhui Province. http://www.stats.gov.cn/tjsj/tjgb/rkpcgb/dfrkpcgb/201202/t20120228_ 30380.html (Accessed on 17 May 2011).

23. Wang J, Wang $P$, Wang $X$, et al. Use and prescription of antibiotics in primary health care settings in China. JAMA Intern Med. 2014;174:1914-20.

24. Harris A, Chandramohan S, Awali RA, et al. Physicians' attitude and knowledge regarding antibiotic use and resistance in ambulatory settings. Am J Infect Control. 2019;47(8):864-8.

25. Langley T, Lacey J, Johnson A, et al. An evaluation of a price transparency intervention for two commonly prescribed medications on total institutional expenditure: a prospective study. Future Healthc J. 2018;5(3):198-202.

26. Ogunleye OO, Fadare JO, Yinka-Ogunleye AF, et al. Determinants of antibiotic prescribing among doctors in a Nigerian urban tertiary hospital. Hosp Pract. 2019;47(1):53-8.

27. Chang Y, Chusri S, Sangthong R, et al. Clinical pattern of antibiotic overuse and misuse in primary healthcare hospitals in the southwest of China. PLoS ONE. 2019;14(6):e0214779.
28. Diao M, Shen X, Cheng J, et al. How patients' experiences of respiratory tract infections affect healthcare-seeking and antibiotic use: insights from a cross-sectional survey in rural Anhui, China. BMJ Open. 2018;8(2):e019492.

29. O'Connor R, O'Doherty J, O'Regan A, et al. Medical management of acute upper respiratory infections in an urban primary care out-of-hours facility: cross-sectional study of patient presentations and expectations. BMJ Open. 2019;9(2):e025396.

30. China National Health commission. The report of antimicrobial management and bacterial drug resistance status in China. Beijing: Peking Union Medical University Press; 2018.

31. David G, Kerenza H, Daniel F, et al. Adherence-adjusted estimates of benefits and harms from treatment with amoxicillin for LRTI: secondary analysis of a 12-country randomised placebo-controlled trial using randomisation-based efficacy estimators. BMJ Open. 2015;5(3):e006160.

32. Thornton HV, Turner KM, Harrison S, et al. Assessing the potential of upper respiratory tract point-of-care testing: a systematic review of the prognostic significance of upper respiratory tract microbes. Clin Microbiol Infect. 2019;25(11):1339-46.

\section{Publisher's Note}

Springer Nature remains neutral with regard to jurisdictional claims in published maps and institutional affiliations.
Ready to submit your research? Choose BMC and benefit from:

- fast, convenient online submission

- thorough peer review by experienced researchers in your field

- rapid publication on acceptance

- support for research data, including large and complex data types

- gold Open Access which fosters wider collaboration and increased citations

- maximum visibility for your research: over 100M website views per year

At BMC, research is always in progress.

Learn more biomedcentral.com/submissions 